\title{
The iMeMex Dataspace Management System: Architecture, Concepts, and Lessons Learned
}

\author{
Jens Dittrich \\ Saarland University
}

\begin{abstract}
The iMeMex Project was one of the first systems trying to build a so-called dataspace management system. This tutorial presents the core concepts of iMeMex. We discuss system design concepts, dataspace modelling, dataspace indexing, dataspace query processing, and pay-as-you-go information integration. We will present some important lessons learned from this project and also discuss ongoing and open research challenges.
\end{abstract}

\section{CV}

Jens Dittrich is an Associate Professor of Computer Science at Saarland University (Germany). He received his Diploma and PhD from the University of Marburg (Germany). He held positions at SAP AG (Germany) and ETH Zurich (Switzerland). His research interests are in the area of information systems and databases, and, in particular, new system architectures for information management, indexing, data warehousing, and main memory databases.

Web-site: http://infosys.cs.uni-sb.de

\section{Slides and Other Material}

Slides will be made available electronically 11 after the talk. An accompanying article will appear in the Data Engineering Bulletin, special issue on "New Avenues in Search" in June 2009.

${ }^{1}$ http://infosys.cs.uni-sb.de/jensdittrich.php 